\title{
Microscopic dynamics in molten Ni: Experimental scrutiny of embedded-atom-potential simulations
}

\author{
M. D. Ruiz-Martín, M. Jiménez-Ruiz, and M. Plazanet \\ Institute Laue Langevin, 6 Rue Jules Horowitz, F-38042-Grenoble Cedex 9, France
}

F. J. Bermejo, R. Fernández-Perea, and C. Cabrillo*

Instituto de Estructura de la Materia, CSIC, and Departamento Electricidad y Electrónica-Unidad Asociada CSIC, Facultad de Ciencia

y Tecnología, Universidad del País Vasco/EHU, P.O. Box 644, E-48080-Bilbao, Spain

(Received 30 January 2007; revised manuscript received 12 March 2007; published 5 June 2007)

\begin{abstract}
The stochastic dynamics in molten nickel is studied by neutron scattering. The quasielastic spectrum shows two distinct components having disparate linewidths. The wave-vector dependence of the narrow component is shown to arise from incoherent scattering at low momentum transfers. In turn, the spectral half-width of the wider component shows a modulation with wave vector characteristic of coherent quasielastic scattering. The analysis of both components provides direct experimental estimates for the self-diffusion coefficient, as well as the effective particle diameter. The experimental data are then used to validate computer simulation results which are derived using an embedded-atom potential. Such results are also employed to explore regions in frequency-wave-vector space not easily amenable to experiment. In addition, simulation results are also compared with data pertaining to the collective motions. Such an exercise reveals the need to develop a further level in the memory function expansion of the coherent dynamic structure factor. The implications of such findings for our current understanding of the dynamics of liquid metals are finally assessed.
\end{abstract}

DOI: 10.1103/PhysRevB.75.224202

PACS number(s): 66.10.-x, 66.20.+d, 63.20.Ls

\section{INTRODUCTION}

Studies on the transport properties of molten transitionmetal elements have mostly remained within the realm of computer simulations ${ }^{1}$ due to stringent conditions imposed by sample containment requirements. ${ }^{2}$ Such a dearth of reliable experimental data contrasts with the technological or geophysical $^{3}$ importance of such materials. In fact, knowledge of the transport properties of liquid iron, nickel, and mixtures of both elements with a third light-alloying component such as sulfur or silicon is of fundamental importance for the understanding of the basic physics of many planetary cores, including Earth. A glance at experimental data concerning the perhaps most fundamental transport property such as the shear viscosity of molten Ni serves to exemplify the state of affairs on these matters since the available data set $^{4}$ yields estimates spanning some $60 \%$ around its mean value.

On a more fundamental vein, additional interest in pursuing the studies reported here arises from consideration of liquid metals as the closest physical realization within the microscopic realm of a hard-sphere fluid., ${ }^{5,6}$ The attractiveness of such similitude comes from the availability of closedform expressions for most transport and dynamical properties of hard-sphere or even Lennard-Jones fluids which have been derived from recourse to kinetic theory, ${ }^{7}$ and therefore, studies on liquid metals provide a benchmark to scrutinize the accuracy of such theoretical predictions.

Although a good number of simulations by computer molecular dynamics (MD) on liquid $\mathrm{Ni}$ of varying degrees of sophistication have already appeared, ${ }^{1,8}$ their predicted results have most of the times been compared to structural, mechanical, or macroscopic properties only. Here, we report on a comparison between the computer predictions and prop- erties most sensitive to fine details of atomic interactions such as those pertaining to the atomic dynamics within the microscopic realm. In particular, we explore here the predictive capabilities of MD approaches based on embedded-atom-potentials ${ }^{8}$ (EAMs) versus experimental data derived from neutron scattering. To such an avail, the experiments reported here mostly concern the low-frequency part of the wave-vector-dependent frequency spectrum $S(Q, \omega)$ or dynamic structure factor which can be accessed with rather high resolution in energy transfers by means of cold-neutron spectroscopy. Such a range of frequency is dominated by quasielastic-scattering effects which are produced by Doppler broadening of the incident beam due to its interactions with stochastic particle motions such as spacedependent mass diffusion. Because of the characteristics of neutron interactions with the nuclei of normal abundance $\mathrm{Ni}$, we explore here both single-particle properties which dominate the measured double-differential scattering cross sections for values of the momentum transfers where coherent effects are minimal [i.e., well below those where the static structure factor $S(Q)$ attains relatively large values] and those related to stochastic albeit correlated particle motions. Both kinds of motions represent those executed by a tagged particle which give rise to the incoherent scattering structure factor $S_{i n c}(Q, \omega)$, as well as those giving rise to backflow due to the motion of a given particle which are seen as an interference effect within the coherent $S_{c}(Q, \omega)$ structure factor.

A second issue of interest concerns the part of $S_{c}(Q, \omega)$ that is dominated by finite-frequency excitations, which, for a molten metal such as $\mathrm{Ni}$, exclusively corresponds to acoustic phonons. Although experimental data for such excitation frequencies ${ }^{9}$ were recently compared to simulation results by Jakse et al., the main focus of interest concerns some developments which arose from the analysis of the spectral line 
shape of the complete $S_{c}(Q, \omega)$. In fact, as we show below, proper account of the calculated $S_{c}(Q, \omega)$ requires an extension of the theoretical frameworks which have been shown to be suitable to reproduce data for the molten alkali metals ${ }^{10}$ but fail to account for that on liquid Ni.

In the following sections, we summarily describe the experimental and computational methods, as well as some of the results and a detailed comparison between both. Results derived from the MD simulation are later described and, finally, the relevance of the present findings for our current knowledge on the dynamics of liquid metals within microscopic scales is briefly addressed.

\section{EXPERIMENT}

The experiment was performed on the IN5 cold-neutron chopper spectrometer located at the Institut Laue Langevin (ILL) (Grenoble, France). This instrument offers large flexibility for wavelength selection and chopper speed which permits considerable optimization of the energy range, energy resolution, momentum transfer, and count rate.

The sample, a Ni powder of $99.999 \%$ purity, was held within a flat ceramic container made of $\mathrm{Al}_{2} \mathrm{O}_{3}$ to avoid the chemical reactions that usually occur between the sample and the container when working at high temperatures. Its dimensions were calculated in order to have $\sim 20 \%$ of the incoming neutrons scattered and/or absorbed by the sample plus container, taking into account the values of the neutron scattering and absorption cross sections, $\sigma_{\text {scatt }}=18.5 \mathrm{~b}$ and $\sigma_{a b s}=4.49 \mathrm{~b}$, respectively. The slab container had dimensions of $40 \times 27 \mathrm{~mm}^{2}$ and the wall thickness was $2 \mathrm{~mm}$. The liquid state was achieved by slowly heating the sample up to its melting point into a $\mathrm{Nb}$ furnace designed at ILL. The entire ensemble is under vacuum to prevent its oxidation, at pressures of $\sim 10^{-6}$ mbar. The temperature was measured with two tungsten thermocouples placed in close contact with the cell. In order to minimize the background, the flight path (volume) between the sample and the detectors is filled with argon.

Two sets of measurements were carried out employing two different wavelengths. The first corresponds to $\lambda$ $=5.5 \AA^{-1}$, which gave us access to the region of small $Q$ values where incoherent scattering dominates the doubledifferential scattering cross section. The energy resolution, as measured at the elastic peak position using vanadium foil for this configuration, was a Gaussian of $94 \mu \mathrm{eV}$, full width at half maximum.

The second set of measurements was performed with a higher energy of the incident beam and used a shorter wavelength $\left(\lambda=2.5 \AA^{-1}\right)$. These measurements allowed us to map a wider region of the $(Q, \omega)$ reciprocal space with far less resolution in energy transfers $(0.65 \mathrm{meV})$.

Naturally abundant $\mathrm{Ni}$ is mostly a coherent scatterer having a cross section $\sigma_{c}=13.3 \mathrm{~b}$, while the incoherent scattering yields $\sigma_{\text {inc }}=5.2 \mathrm{~b}$, thus totaling $\sigma_{\text {scatt }}=18.5 \mathrm{~b}$. Both single-particle and collective dynamical properties are of interest here. The former contributes to the incoherentscattering cross section and the latter yields purely coherent scattering. The total cross section $I(Q, \omega)$ would then be composed by a sum of incoherent and coherent dynamic structure factors $S_{\alpha}(Q, \omega)$,

$$
\begin{gathered}
I(Q, \omega)=A\left[\frac{\sigma_{\text {inc }}}{\sigma_{\text {scatt }}} L_{\text {inc }}(Q, \omega)+\frac{\sigma_{c}}{\sigma_{\text {scatt }}} L_{c}(Q, \omega)\right] \otimes R(Q, \omega), \\
L_{\alpha}(Q, \omega)=\frac{\Delta \omega_{\alpha}}{\omega^{2}+\Delta \omega_{\alpha}^{2}},
\end{gathered}
$$

where $A$ is a global normalization constant and $R(Q, \omega)$ stands for the finite instrument resolution function, and the functional form assumed for both components corresponds to Lorentzian function $L_{\alpha}(Q, \omega)$ with half-widths $\Delta \omega_{\alpha}$. As we will show below, an instrumental separation of coherent and incoherent contributions is made possible by the rather disparate values exhibited by both incoherent- and coherentscattering linewidths. At momentum transfers well below that where the static structure factor $S(Q)$ shows its maximum, $Q_{p}=3.1 \AA^{-1},{ }^{13}$ the scattering is dominated by the incoherent cross section.

The spectral intensity shown in Fig. 1 arises from incoherent-scattering effects, with the exception of a small, nearly $Q$-independent background term attributable to mostly coherent effects. Within the range of momentum transfers where incoherent scattering dominates, the spectral response is described very well by a single Lorentzian with halfwidths $\Delta \omega_{\text {inc }}$ and peak intensities $S_{\text {inc }}(Q, 0)$, which are also shown in Fig. 1. The results show that the asymptotic $\Delta \omega_{i n c} \propto Q^{2}$ (i.e., Fickian) behavior is approximately followed by the linewidths up to relatively large momentum transfers $Q \approx 1.5 \AA^{-1}$ and the same applies to the intensity that follows $S(Q, 0) \propto Q^{-2}$. From such data, an estimate for the apparent self-diffusion coefficient is derived since the expression $\Delta \omega_{i n c}=D_{s} Q^{2}$ applies and yields $D_{s}=0.28 \mathrm{meV} \AA^{2}$. This figure is to be compared to computational estimates that range between $D_{s}=0.1672 \mathrm{meV} \AA^{2}$ and $D_{s}=0.3403 \mathrm{meV} \AA^{2} .1,8$ Also, the dependence with wave vector of the intensity at the peak maximum $S(Q, \omega=0)$ follows an apparently hydrodynamic dependence according to $S(Q, \omega=0) \propto 1 / \pi D_{s} Q^{2}$. Some deviations from such long-wavelength behavior are expected due to coupling between particle diffusion and the collective modes and are discussed below with the aid of computer simulation results.

The measurements employing the shorter wavelength gave us access to a more extended range of frequencies. The data shown in Fig. 2 have been analyzed on the grounds set by Eq. (1) using the values derived for the incoherent component that have been discussed above as a constraint. A sample of spectra corresponding to wave vectors comprising the maximum of the structure factor $S(Q)$ is shown in Fig. 2, together with the results depicting the variation with momentum transfer of the coherent linewidth, as well as its intensity.

As shown in Fig. 2, the $Q$ dependence of the integrated intensity within this spectral range follows the oscillations present in $S(Q)$. This proves that this wider quasielastic component is dominated by coherent effects. As derived from kinetic theory ${ }^{11}$ and proven against experimental data in 


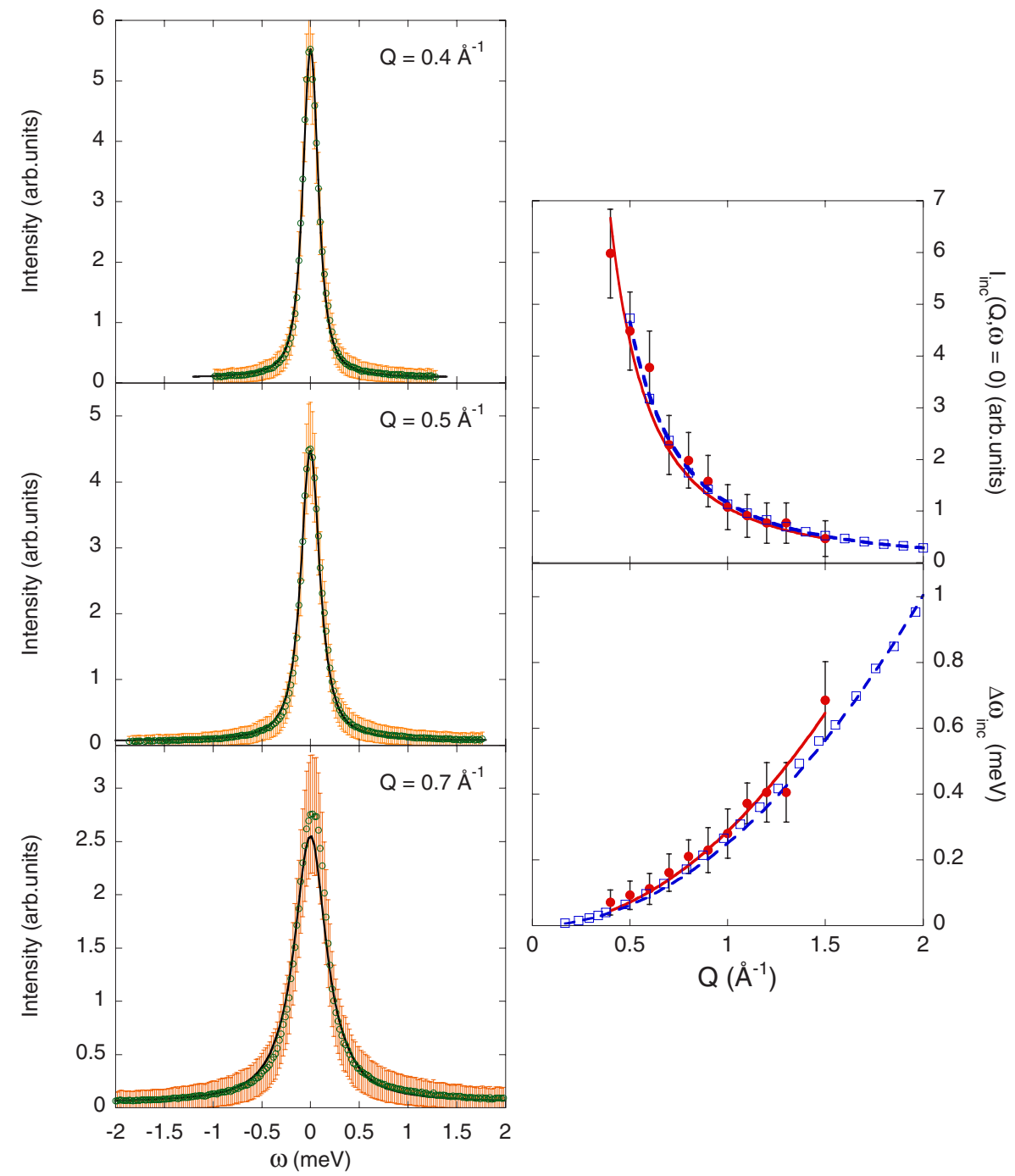

FIG. 1. (Color online) The left-hand side shows spectra as measured using the lowest incident energy. The right-hand side depicts the dependence with momentum transfers of the linewidths and peak intensities. Open symbols depict computer simulation data which are considered below. Lines are best fits to either experimental or computer simulation data. some other liquid metals, ${ }^{12}$ an approximate expression to describe the half-width of the coherent quasielastic spectrum for wave vectors comprising $Q_{p}$, where the static $S(Q)$ shows its maximum, is given by

$$
\Delta \omega_{c}(Q)=\frac{D_{s} Q^{2}}{S(Q)\left[1-j_{0}\left(Q r_{m}\right)+2 j_{2}\left(Q r_{m}\right)\right]} .
$$

In addition, as shown in Ref. 12, good results are obtained if the particle self-diffusion coefficient is set to its experimental value rather than that given by the Enskog prescription, as initially proposed. ${ }^{11}$ The parameter $r_{m}$ is defined by the value where the radial distribution shows its first maximum, and experimental data for $S(Q)$ have been taken from Ref. 13. Finally, $j_{x}()$ are spherical Bessel functions. A comparison between the experimental values for the linewidths about $Q_{p}$ and those calculated using Eq. (3) using the value for $D_{s}$ derived here ${ }^{1}$ reveals that the linewidths can be accounted for in terms of Eq. (3) which, in other words, means that no contributions different from those of structural nature (i.e., thermal diffusivity) need to be called upon to explain such a spectral component.

\section{COMPUTER SIMULATIONS}

The simulations were performed for an ensemble of 4000 atoms close to melting $\left(T_{m}=1728.15 \mathrm{~K}\right)$ at $T=1800 \mathrm{~K}$. We have employed the EAM potential used by Ogando Arregui et $a l .{ }^{1}$ that did adequately reproduce the structure and thermodynamics of both the hot solid and the liquid. An initial fcc crystal configuration was melted at high temperature and equilibrated under NPT conditions, yielding a final number density matching the experimental estimate of $\rho=0.078 \AA^{-3}$. Production runs of $82 \mathrm{ps}$ were carried out in order to compute the properties of interest.

The $S_{c}(Q, \omega)$ dynamic structure factors to be compared with those derived from coherent neutron scattering of the liquid were calculated as an average over 80 different configurations for an interval $0.128 \AA^{-1} \leqslant Q \leqslant 3.128 \AA^{-1}$.

Four different runs were needed to obtain accurate estimates of the $S_{\text {inc }}(Q, \omega)$ self-dynamic structure factors. These were calculated over a wide range in $Q$ stretching from $Q$ $=0.128 \AA^{-1}$ to $Q=9.707 \AA^{-1}$. For those runs with wave vectors $Q<0.35 \AA^{-1}$, the simulation time was extended up to 829.6 ps to allow for a complete decay of the intermediate scattering function. 


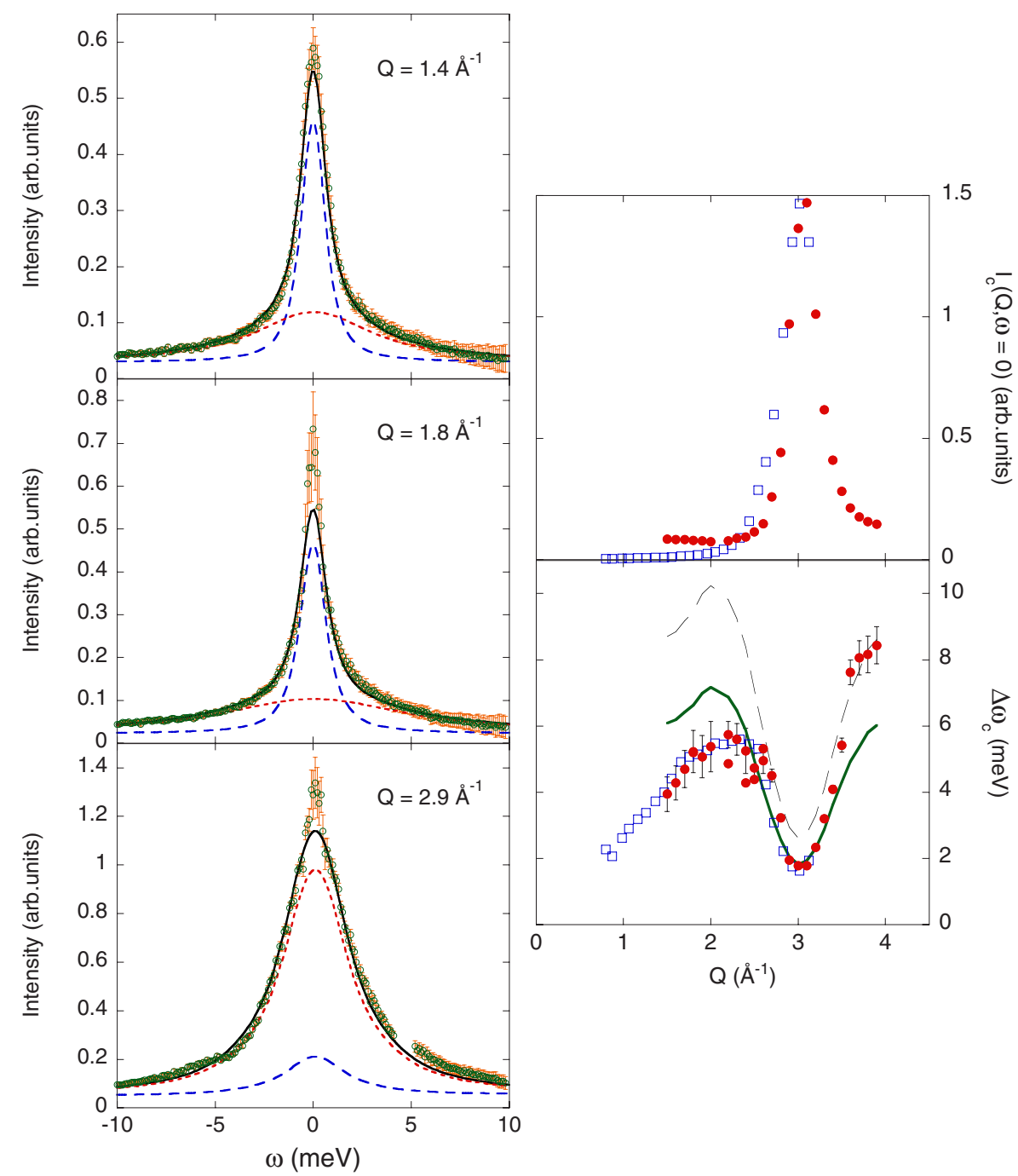

FIG. 2. (Color online) The left-hand side shows spectra as measured using the higher incident energy. Dotted lines depict the coherentscattering contribution, while dashed lines account for the incoherent-scattering contribution described above. The right-hand side depicts the dependence with momentum transfers of the linewidths and peak intensities. Solid symbols show experimental results while open symbols depict data derived from computer simulations that are discussed below. The two curves comparing experimental and calculated values for $\Delta \omega_{c}(Q)$ depict the functions calculated using our experimental estimate for the self-diffusion coefficient (solid), as well as that calculated using the value given by the revised Enskog theory (see below).

The calculated $S(Q)$ was found to be in good agreement with experimental neutron and $\mathrm{x}$-ray diffraction results ${ }^{13,14}$ as far as the perfect match of the position and width of the main diffraction peak. However, and in common with most published simulation results, the simulation overemphasizes the height of $S(Q)$. On the other hand, the estimate for the selfdiffusion coefficient, as calculated from the long-time limit of the mean-squared displacement (msd) $\left\langle u^{2}\right\rangle$, yields a value of $0.26(3) \mathrm{meV}^{2}$, to be compared with our estimate of $0.28 \mathrm{meV} \AA^{2}$, as well as with that of $0.30 \mathrm{meV} \AA^{2}$ derived from a diffusion equation ${ }^{15}$ based on a hard-sphere theory. What particularly merits some comment concerns the long time required for the msd to achieve the linear regime $(\approx 40 \mathrm{ps})$. In fact, our results show a region of strong ballistic motion attributable to vibratory motions that extends up to about $10 \mathrm{ps}$, followed by an intermediate-time region reflecting a strong coupling between single-particle and vibratory (collective) motions.

\section{RESULTS AND DISCUSSION}

The main aim of the experiments described here was to validate simulation results which provide us with access to regions of momentum and/or energy transfer not easily accessible by experiment. Data already shown for the $Q$ dependence of the incoherent and coherent quasielastic linewidths displayed in Figs. 1 and 2 prove that the simulation is able to reproduce the measurements up to a significant level of accuracy and, on such grounds, we extend the kinematic range explored by experiments solely by the use of simulation data.

\section{A. Single-particle dynamics}

A set of calculated $S_{i n c}(Q, \omega)$ dynamic structure factors is displayed in Fig. 3 on a semilogarithmic scale. A first point to remark here concerns the presence of a noticeable broad inelastic wing that shows a width nearly independent of wave vector. 


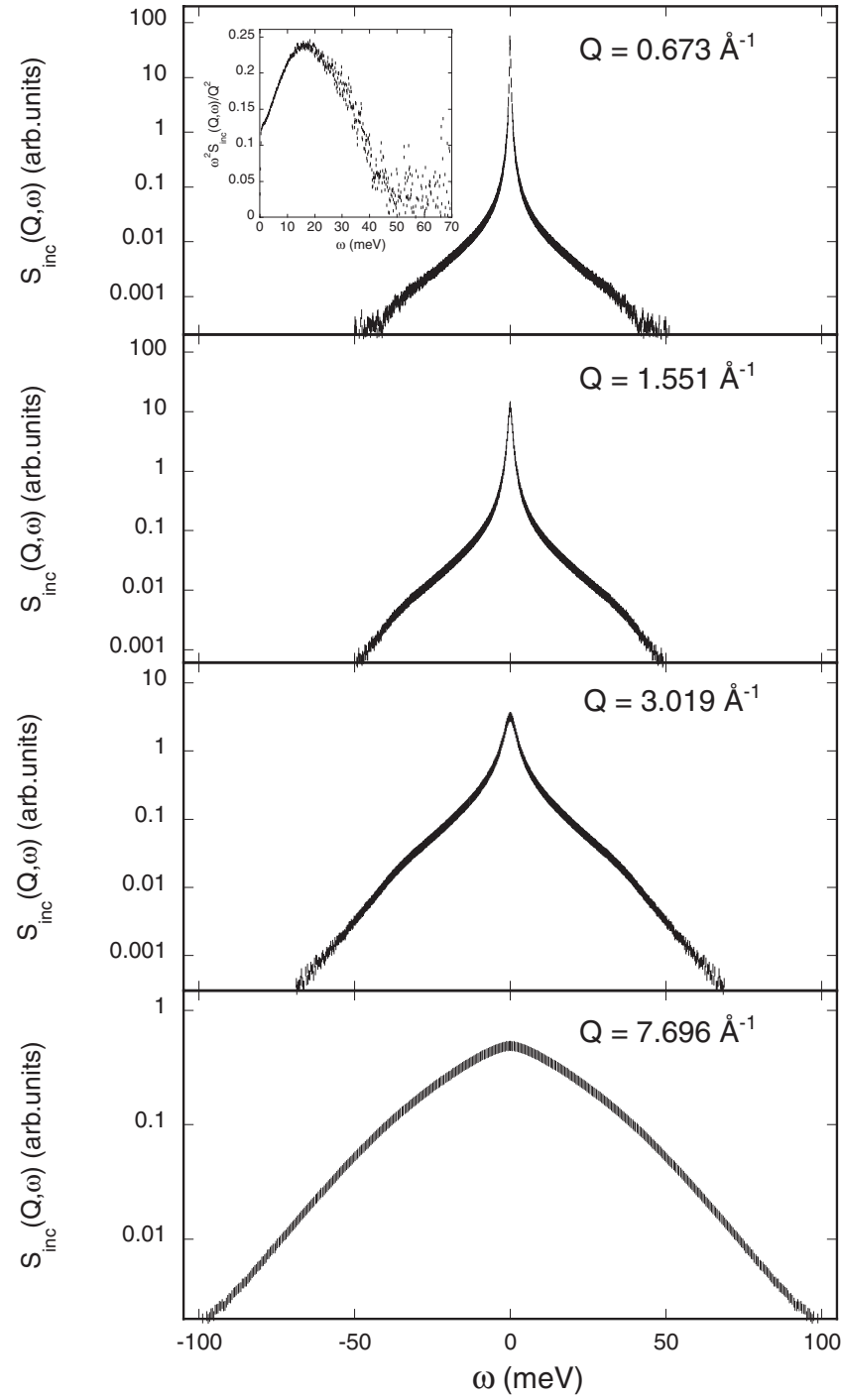

FIG. 3. A sample of self-dynamic structure factors calculated for wave vectors given as insets. The uppermost inset depicts the quantities $\omega^{2} S_{\text {inc }}(Q, \omega) / Q^{2}$ showing the presence of the broad inelastic background.

Such a feature seems to be related to the markedly sharp peak that appears in the Fourier transform of the particle velocity time-autocorrelation function, which is shown in Fig. 4 as the normalized frequency spectrum $Z(\omega)$.

The peak which comes out at some $17.4 \mathrm{meV}$ in $Z(\omega)$ is far better defined here than those seen for other alkali metals such as potassium ${ }^{10}$ and, again in contrast with the alkali metals, it has a significantly lower value than our estimate for the Einstein frequency which yields $30.27 \mathrm{meV}$. Its frequency extent curiously enough matches that for the first peak within the vibrational density of states of room temperature Ni. ${ }^{16}$

From the calculated set of spectra, the peak maxima and linewidth of the central quasielastic component were measured. The first comparison between the experimental and calculated data for the linewidths and amplitudes has already been shown in Fig. 1, which shows that experimental and simulation data are remarkably close. There are, however,

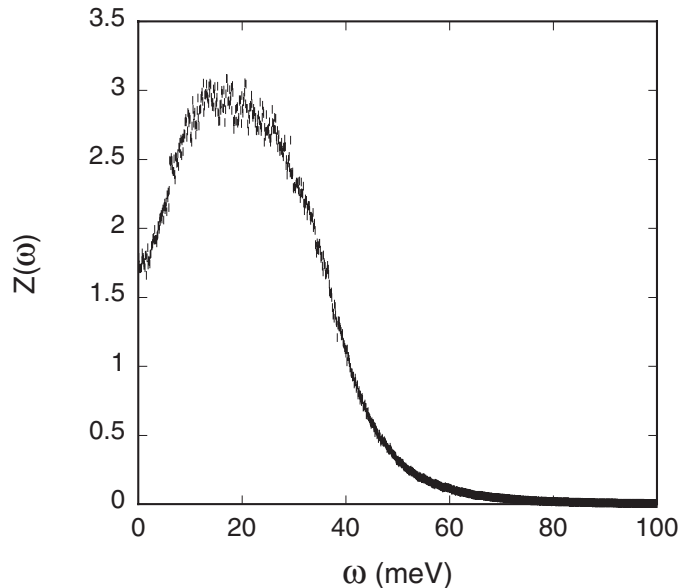

FIG. 4. Normalized frequency spectrum as calculated from the velocity autocorrelation function of the particle velocity $\langle v(0) \cdot v(t)\rangle$.

relevant details involving larger momentum transfers which are difficult to access by experimental means but are easily explorable by simulations. Figure 5 displays the wave-vector dependence of the reduced half-width $\delta(Q)=\Delta \omega_{1 / 2} / D Q^{2}$ and the peak height $S_{\text {inc }}(Q, \omega=0) Q^{2}$.

The reduced linewidth $\delta(Q)$ shows that a small but significant deviation from the Fickian regime can be accounted for in terms of the function $\delta_{c}(Q)=1-b(\rho, T) Q^{2}$, as given by the mode-coupling prediction, ${ }^{17}$ at the lower $Q$ values. The value for the density- and temperature-dependent coefficient

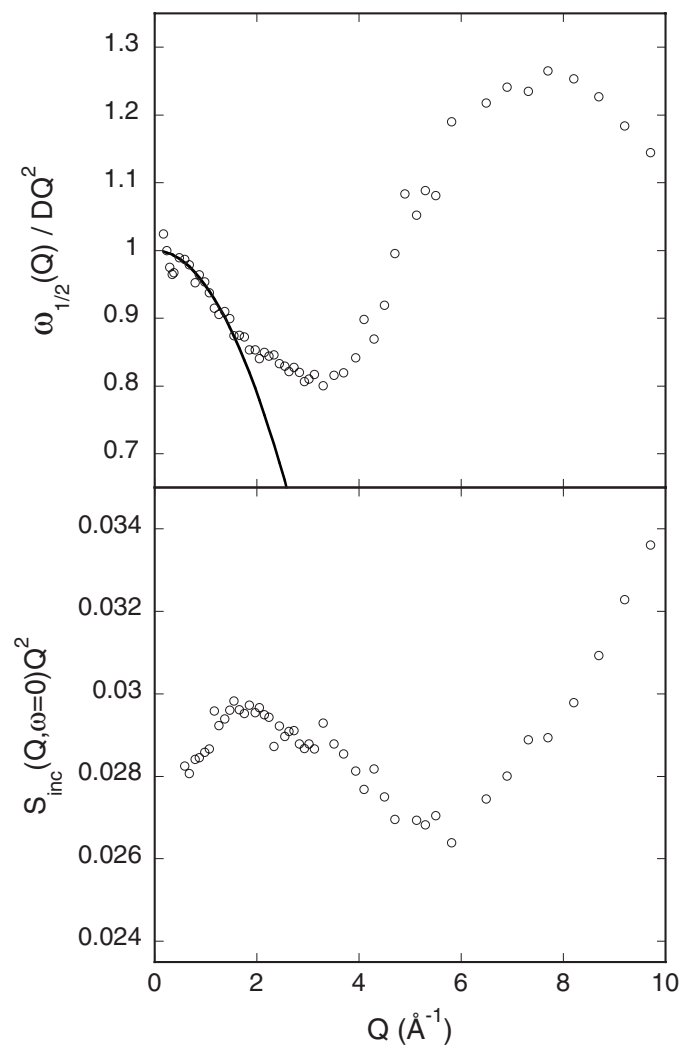

FIG. 5. The upper frame displays the reduced linewidth $\Delta \omega_{1 / 2} / D Q^{2}$ (symbols) and the solid line shows the theoretical prediction (see text). The lower frame shows $S_{\text {inc }}(Q, \omega=0) Q^{2}$. 

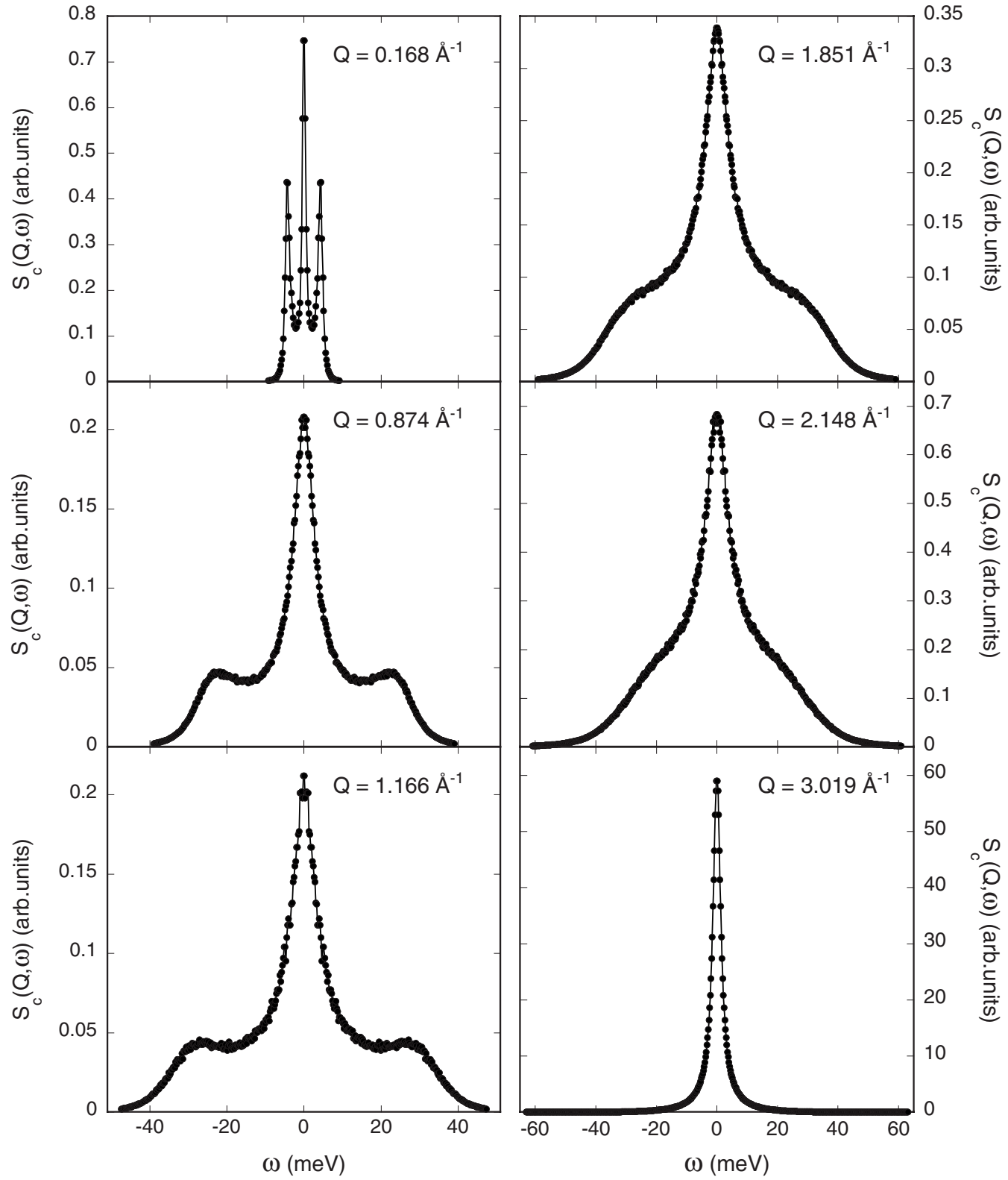

FIG. 6. A set of calculated $S_{c}(Q, \omega)$ spectra spanning wave vectors up to those where $S(Q)$ shows its maximum (symbols). The solid lines are fits to the model described below.

gives $0.05 \AA^{2}$, which compares with that of $0.074 \AA^{2}$ reported for sodium near melting. ${ }^{17}$ Such deviation is interpreted as a retardation effect of mass diffusion due to a strong coupling with longitudinal collective modes. Put into different words, under the present conditions, mass diffusion takes place only after a relatively large lapse of time where liquid cages become unable to maintain collective oscillatory motions.

A marked crossover from a sub-Fickian regime to another with linewidths exceeding the $D Q^{2}$ limit sets in for both the reduced linewidth and amplitude shown in Fig. 5 at wave vectors past that signing the maximum in $S(Q)$. In fact, both quantities show a change in trend, as measured by the shape parameter $B(Q)=2 \Delta \omega_{1 / 2} S_{s}(Q, \omega=0)$, which monitors a departure from the Lorentzian line shape toward a more Gaussian form that becomes dominant at large $Q$. Notice, however, that because of the close-packed arrangement within the liquid, free particle motions (i.e., free gas behavior) are confined to wave vectors well above those explored in this study.

\section{B. Collective dynamics}

A set of $S_{c}(Q, \omega)$ spectra is displayed in Fig. 6 for representative wave vectors. From Fig. 6 , it is readily seen that remarkably well defined excitations persist up to wave vectors above $2 \AA^{-1}$. A first-hand evaluation of the physical frequencies involved in such motions is provided by graphs of maxima of $J(Q, \omega)=\omega^{2} S_{c}(Q, \omega) / Q^{2}$ versus wave vector shown in Fig. 8. Because of well resolved peaks which appear in most of the explored range of wave vectors, the maxima of $J(Q, \omega)$ can be in principle taken as the relevant excitation frequencies. These reach values of $34.5 \mathrm{meV}$ at 
the maxima of the excitation curve that may be favorably compared to those reported from experiment. ${ }^{9}$

In what follows we describe the route followed to analyze such line shapes. We will employ procedures already tested for molten metals. ${ }^{6}$ In particular, our starting point will be that followed by Cabrillo et al. ${ }^{10}$ for the analysis of the spectrum of molten potassium which is based on the generalized Langevin equation formalism. There, the analysis is couched in terms of a model where all the relevant details are encompassed within the $\tilde{M}(Q, \omega)$ memory function so that the spectrum is written as

$$
S_{c}(Q, \omega)=S(Q) \mathfrak{R}[i \omega+\tilde{M}(Q, \omega)]^{-1} .
$$

The simplest form for $\tilde{M}(Q, \omega)$ assumes that viscous damping having a single-relaxation time $\tau$ is the dominant process in the decay of density oscillations since further decay channels are only relevant for far shorter times. Such an approach is referred to in the literature as the "viscoelastic" ansatz which represents a microscopic correlate of the relaxation time $\tau_{v i s c}=\eta / G$, where $\eta$ stands for the shear viscosity and $G$ is the spring constant of the Maxwell element. ${ }^{18}$ Such a single-relaxation time approach was unable to account for the spectrum of molten $\mathrm{K}$ and the same applies here. An improvement able to account for the experimental line shape was implemented by Cabrillo et al. ${ }^{10}$ that extends a further level in the continued fraction expansion of the memory function, so that

$$
\tilde{M}(Q, s)=\omega_{0}^{2}\left[s+\frac{\omega_{1}^{2}-\omega_{0}^{2}}{s+\frac{\omega_{s}^{4}-\omega_{1}^{4}}{\left(\omega_{1}^{2}-\omega_{0}^{2}\right)(s+1 / \tau)}}\right]^{-1},
$$

where $\omega_{0}^{2}$ and $\omega_{l}^{2}$ are the second spectral frequency moment, normalized to $S(Q)$, and the fourth frequency moment, which is defined below, and $\omega_{s}^{4}$ is the sixth frequency moment, which was treated there as a free parameter. Both $\omega_{l}^{2}$ and $\omega_{s}^{2}$ are given in terms of $\omega_{0}^{2}$.

Equation (5) did represent an improvement over the simplest form for $\tilde{M}(Q, \omega)$ but still was unable to reproduce spectra for molten $\mathrm{Ni}$ at low and intermediate $Q$ values especially within the quasielastic region. As an example, Fig. 7 compares the best fits obtained using data given by Eq. (5) as a memory function to data reported here. As can be seen from a glance at Fig. 7, Eq. (5) provides a semiquantitative representation of experimental spectra which cannot account for their line shapes in full.

The reason why Eq. (5) fails to provide a better fit to simulated data is not entirely clear. In terms of macroscopic quantities, the main difference between a molten alkali metal such as $\mathrm{K}$ and liquid Ni concerns the more packed structure of the latter, together with enhanced thermal effects. As a matter of fact, one may expect a larger influence of the effects of thermal conduction in liquid $\mathrm{Ni}$ than those operative in K since thermal conductivity $\kappa(T)$ for transition metals is usually three to five times higher than that for molten alkali metals. Such an effect is, however, counterbalanced by the far larger mass densities and larger specific heat $C_{p}$ which makes the heat diffusion coefficient $D_{t h}=\kappa(T) / C_{p}(T) \rho(T)$ be-
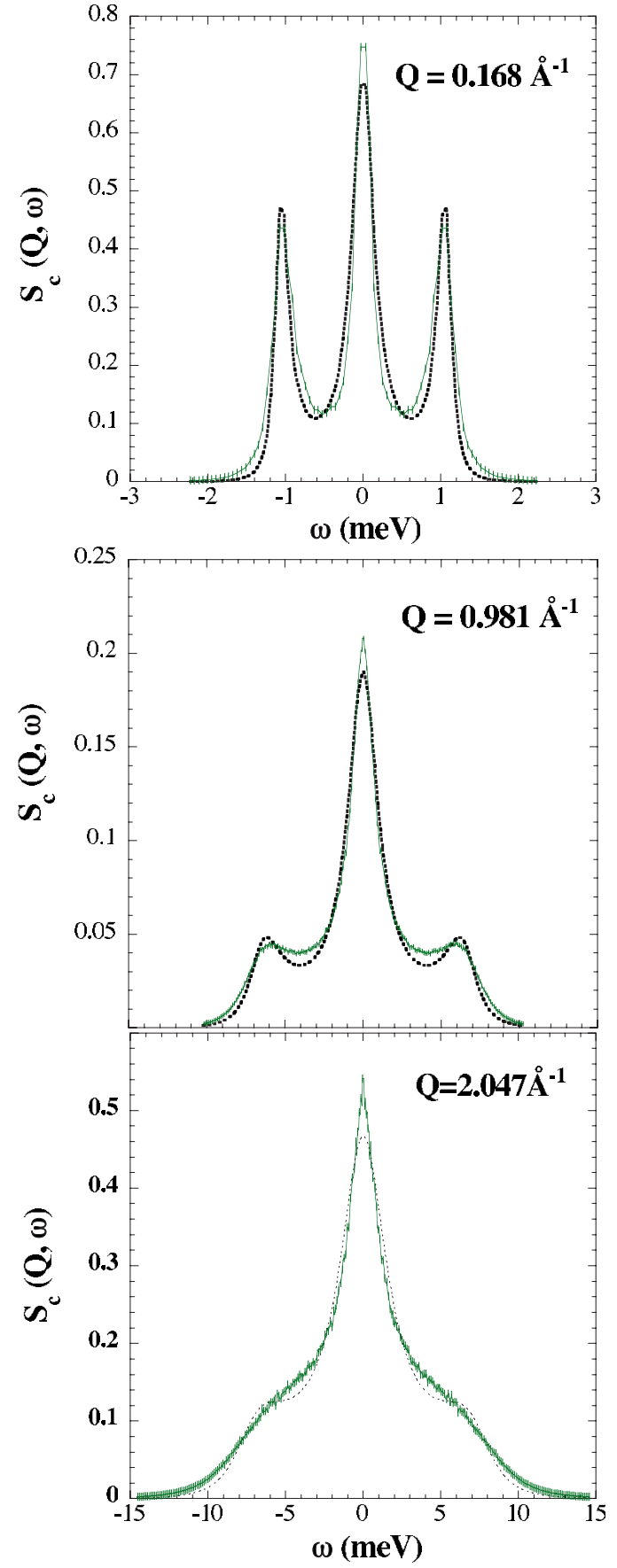

FIG. 7. (Color online) A comparison between calculated spectra for selected values of the momentum transfer (vertical bars) and the best approximation obtained using Eq. (5) as a representation for the memory function (dashes).

come comparable or even smaller than those of molten alkali metals. One also has to take into account that the ratio of specific heats $\gamma$ is here significantly larger than that for molten $\mathrm{K}$ and this would lead us to expect on purely hydrodynamics grounds an additional central component to the spectrum..$^{20}$ At any rate, recent theoretical approaches ${ }^{31}$ couched for the analysis of the dynamics of metallic $(\mathrm{Pb})$ or semimetallic (Bi) liquids make recourse to an extended set of components of the dynamical variable entering the generalized Langevin equation, leading to the appearance of six 
generalized hydrodynamics modes. Similar findings are also reported in a recent reanalysis of experimental data on molten $\mathrm{Al}$ (Ref. 21), which required the use of four-frequency parameters which are needed to account for two-, three-, and four-particle interactions.

Here, we make use of a minimal model able to fit spectra such as those shown in Fig. 6 which makes use of a memory function,

$$
\tilde{M}(Q, s)=\omega_{0}^{2}\left[s+\frac{\left(\omega_{l}^{2}-\omega_{0}^{2} \gamma\right) \tau}{s \tau+1}+\frac{\omega_{0}^{2}(\gamma-1)}{s+\frac{a \tau_{2} Q^{2}}{s \tau_{2}+1}}\right]^{-1},
$$

where $\gamma, \tau$, and $\tau_{2}$ are all wave-vector-dependent parameters, $\omega_{0}^{2}$ is calculated from static quantities as $\omega_{0}^{2}=\frac{Q^{2}}{\beta M S(Q)}, a \tau_{2}$ $=\gamma D_{t h}$ with $D_{t h}$ being the (wave-vector-dependent) thermal diffusivity, and $\omega_{l}^{2}$ is given by

$$
\begin{aligned}
\omega_{l}^{2}= & \frac{3 Q^{2}}{\beta M}+\omega_{E}^{2}\left[1-\frac{3 \sin \left(Q R_{0}\right)}{Q R_{0}}-\frac{6 \cos \left(Q R_{0}\right)}{\left(Q R_{0}\right)^{2}}\right. \\
& \left.+\frac{6 \sin \left(Q R_{0}\right)}{\left(Q R_{0}\right)^{3}}\right],
\end{aligned}
$$

where $\omega_{E}$ plays the role of an Einstein frequency with an optimum (i.e., fitted) value of $30.27 \mathrm{meV}$ and $R_{0}$ stands for a distance which is approximately that corresponding to the main minimum in the interaction potential, and its optimum value was found to be $2.17 \AA$. The derivation of the above equation starts from Eq. (5.196) of Ref. 20 for $\tilde{M}(Q, s)$ which rests within the standard three variable approach. It constitutes the natural and consistent generalization of hydrodynamics treating the three hydrodynamic variables in an equal footing. Fits to Eq. (6) were carried out using a set of five wave-vector-dependent parameters, namely, $D_{t h}, \gamma, \tau, \tau_{2}$, and $a$.

Equation (6) gives rise to five poles in the complex plane, two of which comprise the Brillouin modes. The three additional poles correspond to one of the components of the quasielastic peak arising from correlated motions of a diffusing particle, as described above, a very broad contribution due to thermal diffusion that acts as a background signal, and finally, to a broad contribution centered at zero frequency. The fact that thermal effects, per se, contribute as a background means that the broadening of the Brillouin peaks mostly arises from viscous damping effects.

The model resulting from the use of Eq. (6) is able to represent all the calculated spectra and provides information about the several, wave-vector-dependent quantities. Most of them do have a clear correlate within the hydrodynamic realm. Indeed, the following hydrodynamic limits for the reduced second frequency moment and the thermal contribution are easily verified:

$$
\lim _{Q \rightarrow 0} \omega_{0}^{2}=Q^{2} c_{T}^{2}=Q^{2} \gamma^{-1} c_{s}^{2}
$$

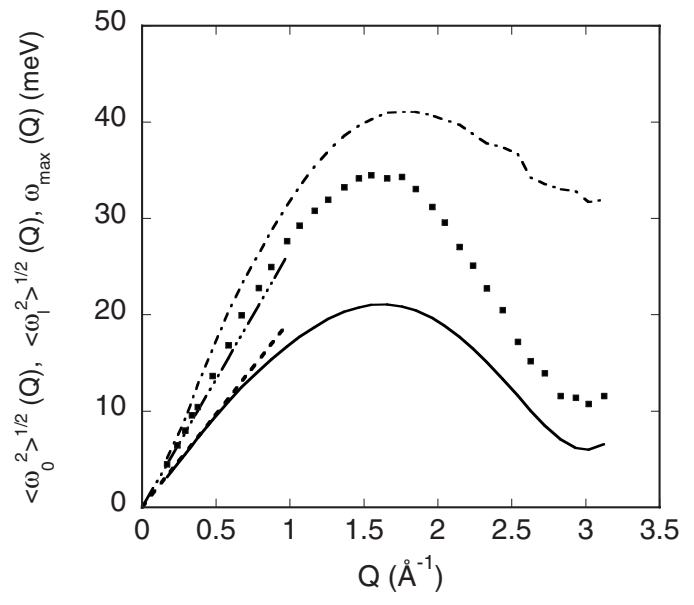

FIG. 8. Maxima of $J(Q, \omega)$ (filled symbols) together with estimates for the second $\left\langle\omega_{0}\right\rangle^{1 / 2}$ (solid line) and fourth $\left\langle\omega_{l}\right\rangle^{1 / 2}$ (dashed) reduced frequency moments. The straight lines give the hydrodynamic dispersion using the tabulated values of the adiabatic sound velocity (Ref. 19) (dash dots), as well as the isothermal value, as calculated using the tabulated value for the ratio of specific heats (Ref. 19).

$$
\lim _{Q \rightarrow 0} a(Q) \tau_{2}(Q)=\gamma D_{t h},
$$

where $c_{s}$ is the adiabatic sound velocity, $\gamma$ represents the ratio between heat capacities, and $D_{t h}$ is the thermal diffusivity.

As seen from Fig. 8, the hydrodynamic isothermal sound velocity $c_{T} Q$ joins $\left\langle\omega_{0}\right\rangle^{1 / 2}(Q)$ at about $0.5 \AA^{-1}$, while maxima of $J(Q, \omega)$ approach the hydrodynamic law calculated for the adiabatic value at the lowest explored wave vector.

Data concerning parameters related to the thermal contributions are displayed in Fig. 9. The estimated values for the thermal diffusivity show an exponential decay with wave vector extrapolating to values within limits set by different experimental observations. Notice, however, that because of difficulties in measuring adiabatically the thermal conductivity at high temperatures, the experimental estimates span a rather large range of values, ${ }^{22}$ and therefore, the closeness of our result for $Q \rightarrow 0$ and the hydrodynamic values cannot be taken as a proof of the accuracy for the calculated quantity.

As regards the $\gamma(Q)$ which becomes a generalized, wavevector-dependent ratio of the specific heats, it shows a rapid decay with wave vector starting from a macroscopic value of 1.88 and then shows a peak at $Q$ values where $S(Q)$ shows its maximum.

\section{CONCLUSIONS AND OUTLOOK}

One of the most significant results derived within the present work concerns the experimental data per se. Data shown in Fig. 1 display a behavior with momentum transfer for the incoherent linewidth, as well as its peak height which do not strongly depart from $\propto Q^{2}$ and $\propto Q^{-2}$ laws which are expected to be followed for an ensemble of hard spheres. ${ }^{23}$ Such a deceptively simple behavior contrasts, however, with 


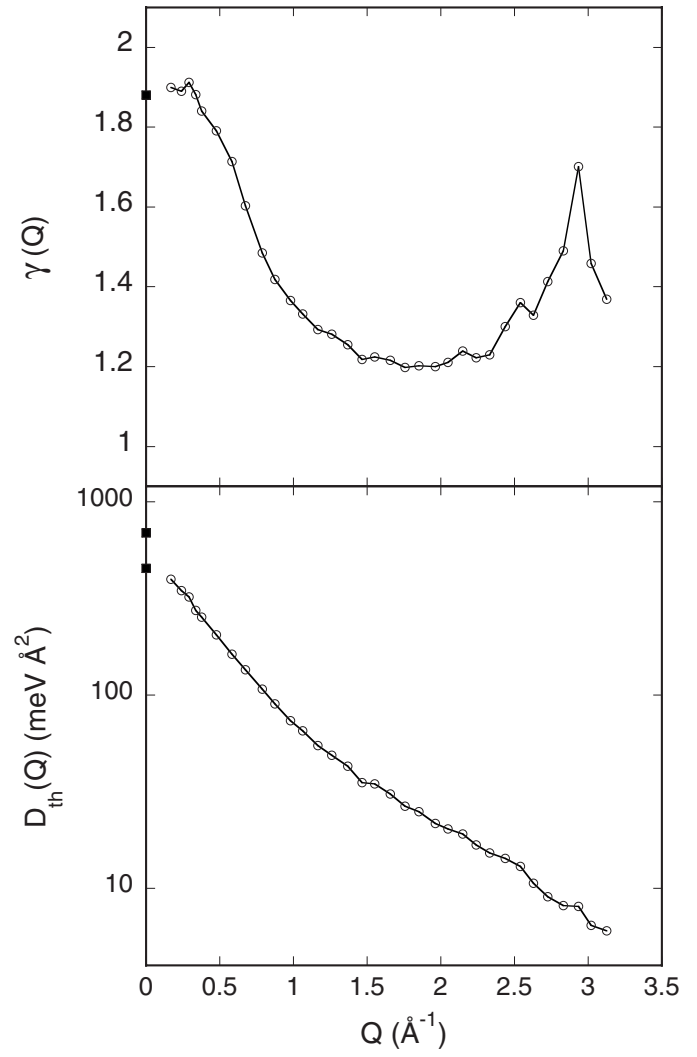

FIG. 9. The upper frame depicts the fitted values found for the wave-vector-dependent ratio of specific heats. The thick symbol on the ordinate axis represents the experimental estimate. The lower frame shows estimates for the generalized, wave-vector-dependent thermal diffusion coefficient. Quantities plotted as solid symbols represent experimental macroscopic estimates. Solid lines are in both cases a guide to the eyes.

the transport properties expected for a hard-sphere fluid of equivalent density. In fact, an estimate for the hard-sphere diffusion coefficient is given by the Enskog expression,

$$
D_{E}=\frac{1}{16} \sqrt{\frac{\pi k_{B} T}{M}} \sqrt[3]{\frac{6}{\pi \rho \eta^{2}}} \frac{(1-\eta)^{3}}{1-\eta / 2}
$$

with the packing fraction defined as $\eta=\pi / 3 \rho \sigma^{3}$. The figure calculated from the above written equation using for the purpose the experimental values for the number density $\rho$ $=0.08 \AA^{-3}$, the atomic mass, and a value for the particle diameter set to that given by the experimental value of the radial distribution at contact, ${ }^{13,24} \sigma=1.88 \AA$, yields $D_{E}$ $=0.75 \AA^{2} \mathrm{meV}$. If $\sigma$ is set by the condition $Q_{p} \sigma=2 \pi$ so that the position of the maxima of the structure factors of molten $\mathrm{Ni}$ and that for an equivalent hard-sphere fluid match, we still get $0.41 \AA^{2} \mathrm{meV}$, which is to be compared to our current experimental value of $D_{s}=0.28 \AA^{2} \mathrm{meV}$. The ratio $D / D_{E}$ $=0.68$ thus provides a first-hand measure on how singleparticle dynamics compare to that of a hard-sphere liquid. To quantitatively estimate the departure from idealized hardsphere behavior, we have evaluated the strength of modecoupling effects which predict that at moderately low values of momentum transfer, the linewidth and peak height of $S_{s}(Q, \omega)$ should vary as ${ }^{25}$

$$
\begin{gathered}
\Delta \omega(Q)=D\left[Q^{2}-b Q^{3}+\mathcal{O}\left(Q^{3 / 2}\right)\right], \\
S_{\text {inc }}(Q, \omega=0)=\frac{1}{\pi D Q^{2}}\left[1+a Q^{-1}+\mathcal{O}\left(Q^{3 / 2}\right)\right] .
\end{gathered}
$$

The two parameters governing the departure from Fickian behavior, namely, $b$ and $a$, can be explicitly calculated from macroscopic properties, as well as from the value of the selfdiffusion coefficient. Their values $b=0.023 \AA$ and $a$ $=0.056 \AA$ are about one-half of those reported for liquid alkali metals where mode-coupling effects are known to be strong. Stronger deviations are also expected for larger values of the momentum transfer, as seen above, as well as demonstrated by previous studies on molten alkali metals. ${ }^{26}$

The comparison of experimental data concerning the coherent linewidth of the quasielastic spectrum to that predicted by Eq. (3) shows that such spectral width for wave vectors close to $Q_{p}$ is mostly attributable to phenomena involving the decay of density fluctuations via self-diffusion processes, as originally proposed in Ref. 11 . Taking it at its face value, this could be taken as an indication of the absence of any significant contribution from thermal diffusion effects arising from either electronic or ionic conduction, a topic which received some attention recently. ${ }^{27}$ Notice, however, that as shown in Ref. 28, the shape of the curve giving the $Q$ dependence of this coherent linewidth for wave vectors is better reproduced if thermal effects are also accounted for.

In terms of characteristic relaxation times which could be attributed to structural relaxation effects, the quasielastic coherent linewidths for wave vectors in the vicinity of $Q_{p}$ correspond to processes taking place within the range of some $0.8-2$ ps.

The need to extend the memory function to account for the line shape of $S_{c}(Q, \omega)$ in full arises from the presence in such quantity of a broad quasielastic component, in addition to that experimentally explored in the present study. Its physical soundness is ascertained here by the presence of a component sharing the same characteristics within the single-particle spectra comprised within $S_{\text {inc }}(Q, \omega)$. Within the framework of Eq. (6), it corresponds to a purely imaginary pole which is clearly distinct from that attributable to thermal diffusion effects since its reaches frequencies up to some $15 \mathrm{meV}$ at $Q \approx Q_{p} / 2$ that are more than 1 order of magnitude smaller than those spanned by the component attributable to a wave-vector-dependent thermal diffusion coefficient shown in Fig. 9. The precise physical origin of such a feature cannot be ascertained at present.

To provide an explicit comparison between the present simulation results concerning $S_{c}(Q, \omega)$ and experimental neutron inelastic scattering data reported in Ref. 9, Fig. 10 depicts a comparison between the estimated excitation frequencies as inferred from the data using for the purpose a conventional damped harmonic oscillator (DHO) model defined by a renormalized frequency $\omega_{\text {osc }}(Q)$, a linewidth $\Gamma_{o s c}(Q)$, and a central quasielastic line defined in terms of its 


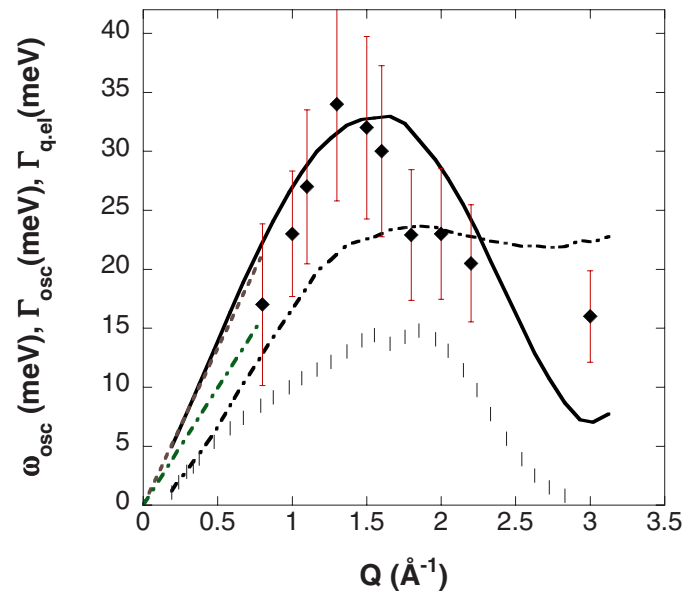

FIG. 10. (Color online) Filled symbols depict the estimates for the experimental frequencies given in Ref. 9. Solid and dash-dot lines show $\Gamma_{o s c}(Q)$ and $\omega_{\text {osc }}(Q)$ derived from fits of a DHO model to $S_{c}(Q, \omega)$. The vertical bars depict the estimated width of the quasielastic component. The dashed and dash-dot straight lines depict the linear hydrodynamic dispersion corresponding to adiabatic and isothermal sound speeds, respectively.

linewidth $\Gamma_{q . e l}(Q)$. In turn, the simulation data were analyzed using the same approach which employs a single quasielastic component.

The comparison depicted in Fig. 10 shows a reasonable agreement between the excitation frequencies obtained from simulation and experimental means. Accounting for the limited accuracy of the neutron scattering data, the experimental points at low momentum transfers seem to lie closer to the hydrodynamic dispersion law rather than that to the adiabatic. Such a result would be expected on the grounds of the large thermal conductivity of molten $\mathrm{Ni}$, together with the high frequencies of the neutron probe, ${ }^{29}$ which make sound propagation isothermal rather than adiabatic. On the other hand, the discrepancy between experimental and simulation data can also be attributed in part to some effects already commented on by some authors ${ }^{8}$ which show that most EAM approaches usually lead to some overestimation of the elastic properties.

The width of the quasielastic component $\Gamma_{q . e l}(Q)$ becomes significantly larger than that explored by experiment using higher resolution in energy transfers, and therefore cannot be attributed to mass-diffusion processes. It basically gives the same values for the linewidth as one of the purely imaginary poles in Eq. (6).

The detailed analysis of $S_{c}(Q, \omega)$ shows that the structure factor significantly departs from the linearized hydrodynamic approximations usually employed to analyze experimental data, such as DHO models. Our present findings show a clear need to go beyond the two-relaxation time approach which seems to account for the dynamics of molten alkali metals reasonably well. ${ }^{6}$ The results come into line with predictions made from some theoretical approaches ${ }^{31}$ which show that the number of components needed to describe in detail the shape of the dynamic structure factor for a molten metal significantly exceeds that of three peaks given by the linearized hydrodynamic prescription. In fact, the predictions made on the grounds of the theoretical framework just referred to portray the presence of two purely relaxing (i.e., quasielastic) kinetic modes in addition to propagating excitations. Put into different words, the present results emphasize the need to account for couplings between structural and thermal degrees of freedom which are deemed to be especially relevant for a molten metal such as $\mathrm{Ni}$.

In summary, the concurrent use of experimental and computer simulation methods to explore the atomic dynamics in this molten transition metal shows that it can only be qualitatively related to that shown by a hard-sphere liquid. Previous attempts to relate the transport coefficients of liquid transition metals to appropriate hard-sphere systems ${ }^{30}$ have to rely on some uncontrolled assumptions to choose the relevant parameters such as the hard-sphere diameter and the packing fraction.

Finally, it goes without saying that further progress in the field is now expected mostly due to the advent of levitation techniques ${ }^{32,33}$ which have been recently employed with success to study the collective dynamics of molten $\mathrm{Ti}^{34}$
*Electronic address: jbermejo@we.lc.ehu.es

${ }^{1}$ B. Sadigh and G. Grimvall, Phys. Rev. B 54, 15742 (1996); G. M. Bhuiyan, M. Silbert, and M. J. Stott, ibid. 53, 636 (1996); D. Alfe and M. J. Gillan, Phys. Rev. Lett. 81, 5161 (1998); M. M. G. Alemany, O Dieguez, C. Rey, and L. J. Gallego, Phys. Rev. B 60, 9208 (1999); F. J. Cherne, M. I. Baskes, and P. A. Deymier, ibid. 65, 024209 (2001); E. Ogando Arregui, M. Caro, and A. Caro, ibid. 66, 054201 (2002); Vocadlo, D. Alfe, M. J. Gillan, and G. D. Price, Phys. Earth Planet. Inter. 140, 101 (2003); L. Koci, A. B. Belonoshko, and R. Ahuja, Phys. Rev. B 73, 224113 (2006).

${ }^{2}$ R. S. Hixson, M. A. Winkler, and M. L. Hodgdon, Phys. Rev. B 42, 6485 (1990).

${ }^{3}$ H. Jeffreys, The Earth (Cambridge University Press, New York 1929), p. 265; P. M. Nasch, M. N. Manghnani, and R. A. Secco,
Science 277, 219 (1997); D. E. Smylie, ibid. 284, 461 (1999); S. C. Singh, M. A. J. Taylor, and J. P. Montagner, ibid. 287, 2471 (2000).

${ }^{4}$ T. IIda and R. I. L. Guthrie, The Physical Properties of Liquid Metals (Oxford Science, Oxford, UK, 1988), Chap. 6, p. 147.

${ }^{5}$ N. H. March, Liquid Metals, (Cambridge University Press, Cambridge, UK, 1990), p. 82.

${ }^{6}$ T. Scopigno, G. Ruocco, and F. Sette, Rev. Mod. Phys. 77, 881 (2005).

${ }^{7}$ G. F. Mazenko, Phys. Rev. A 7, 209 (1973); H. W. Jackson, ibid. 9, 964 (1974); D. Forster, ibid. 9, 943 (1974); G. F. Mazenko, ibid. 9, 360 (1974); M. S. Jhon and D. Forster, ibid. 12, 254 (1975); W. Götze and A. Zippelius, ibid. 14, 1842 (1976); R. C. Desai and R. Kapral, ibid. 17, 477 (1978); L. Sjogren, ibid. 22, 2866 (1980); W. E. Alley, B. J. Alder, and S. Yip, ibid. 27, 3174 
(1983); E. G. D. Cohen, Physica B \& C 127, 282B (1984); W. Goetze, Phys. Rev. A 14, 1842 (1976); J. Sjögren, J. Phys. C 12, 4369 (1979); T. R. Kirkpatrick, Phys. Rev. A 32, 3130 (1985); J. Piasecki and G. Szamel, Phys. Rev. Lett. 60, 1685 (1988); J. Piasecki and G. Szamel, Phys. Rev. A 38, 2124 (1988); F. Perrot, ibid. 42, 4871 (1990); J. W. Dufty, A. Santos, and J. J. Brey, Phys. Rev. Lett. 77, 1270 (1996); N. Mattor and S. E. Parker, ibid. 79, 3419 (1997); M. V. Tokarchuk, I. P. Omelyan, and A. E. Kobryn, Phys. Rev. E 62, 8021 (2000); A. C. Branka and D. M. Heyes, ibid. 69, 021202 (2004); K. M. Dyer, B. M. Pettitt, and G. Stell, J. Chem. Phys. 126, 034502 (2007).

${ }^{8}$ S. M. Foiles, Phys. Rev. B 32, 3409 (1985); S. M. Foiles and J. B. Adams, ibid. 40, 5909 (1989); J. M. Holender, ibid. 41, 8054 (1990); J. Mei and J. W. Davenport, ibid. 42, 9682 (1990); A. Posada-Amarillas and I. L. Garzón, ibid. 53, 8363 (1996); M. M. G. Alemany, C. Rey, and L. J. Gallego, ibid. 58, 685 (1998); M. Asta, D. Morgan, J. J. Hoyt, B. Sadigh, J. D. Althoff, D. de Fontaine, and S. M. Foiles, ibid. 59, 14271 (1999); E. UruutiaBañuelos, A. Posada-Amarillas, and I. L. Garzón, ibid. 66, 144205 (2002); Edmund B. Webb III and G. S. Grest, Phys. Rev. Lett. 86, 2066 (2001); D. Y. Sun, M. Asta, and J. J. Hoyt, Phys. Rev. B 69, 024108 (2004); N. Jakse and A. Pasturel, J. Chem. Phys. 123, 244512 (2005).

${ }^{9}$ F. J. Bermejo, M. L. Saboungi, D. L. Price, M. Alvarez, B. Roessli, C. Cabrillo, and A. Ivanov, Phys. Rev. Lett. 85, 106 (2000).

${ }^{10}$ C. Cabrillo F. J. Bermejo, M. Alvarez, P. Verkerk, A. Maira-Vidal, S. M. Bennington, and D. Martin, Phys. Rev. Lett. 89, 075508 (2002); T. Scopigno, U. Balucani, G. Ruocco, and F. Sette, Phys. Rev. E 65, 031205 (2002); T. Scopigno, U. Balucani, G. Ruocco, and F. Sette, J. Phys.: Condens. Matter 16, S309 (2004); H. Sinn, F. Sette, U. Bergmann, Ch. Halcoussis, M. Krisch, R. Verbeni, and E. Burkel, Phys. Rev. Lett. 78, 1715 (1997); J. R. D. Copley and J. M. Rowe, ibid.. 32, 49 (1974); T. Bodensteiner, Chr. Morkel, W. Gläser, and B. Dorner, Phys. Rev. A 45, 5709 (1992).

${ }^{11}$ E. G. D. Cohen, P. Westerhuijs, and I. M. de Schepper, Phys. Rev. Lett. 59, 2872 (1987); J. J. van Loef and E. G. D. Cohen, Phys. Rev. B 39, 4715 (1989).

${ }^{12}$ F. J. Bermejo, I. Bustinduy, S. J. Levett, J. W. Taylor, R. Fernandez-Perea, and C. Cabrillo, Phys. Rev. B 72, 104103 (2005); F. J. Bermejo, I. Bustinduy, C. Cabrillo, S. J. Levett, and J. W. Taylor, Phys. Rev. Lett. 95, 269601 (2005); T. Scopigno, R. Di Leonardo, L. Comez, A. Q. R. Baron, D. Fioretto, and G. Ruocco, ibid. 95, 269602 (2005); T. Scopigno, R. Di Leonardo, L. Comez, A. Q. R. Baron, D. Fioretto, and G. Ruocco, ibid. 94, 155301 (2005).

${ }^{13}$ O. J. Eder, E. Erdpresser, B. Kunsche, H. Stiller, M. Suda, and P. Winzierl, J. Phys. F: Met. Phys. 9, 1215 (1971).
${ }^{14}$ Y. Waseda and M. Ohtani, Phys. Status Solidi B 62, 535 (1974).

${ }^{15}$ The Physical Properties of Liquid Metals (Ref. 4), p. 105.

${ }^{16}$ R. J. Birgeneau, J. Cords, G. Dolling, and A. D. W. Woods, Phys. Rev. 136, A1359 (1964).

${ }^{17}$ C. Morkel and W. C. Pilgrim, J. Non-Cryst. Solids 312-314, 128 (2002); A. Stangl, C. Morkel, U. Balucani, and A. Torcini, ibid. 205-207, 402 (1996).

${ }^{18}$ D. D. Joseph, Fluid Dynamics of Viscoelastic Liquids, Springer Series in Applied Mathematical Sciences Vol. 84 (SpringerVerlag, New York, 1990), p. 1.

${ }^{19}$ The Physical Properties of Liquid Metals, (Ref. 4), p. 105.

${ }^{20} \mathrm{~S}$. W. Lovesey, Theory of Neutron Scattering in Condensed Matter (Oxford University Press, Oxford 1984), Vol. 1, Chap. 6, p. 210.

${ }^{21}$ A. V. Mokshin, R. M. Yulmetyev, R. M. Khusnutdinov, and P. Hänggi, J. Exp. Theor. Phys. 103, 841 (2006); T. Scopigno, U. Balucani, G. Ruocco, and F. Sette, Phys. Rev. E 65, 031205 (2001).

${ }^{22}$ G. Pottlacher, J. Non-Cryst. Solids 250-252, 177 (1999); T. Nishi, H. Shibata, H. Ohta, and Y. Waseda, Metall. Mater. Trans. A 34A, 2801 (2003).

${ }^{23}$ W. E. Alley and B. J. Alder, Phys. Rev. A 27, 3158 (1983).

${ }^{24}$ The Physical Properties of Liquid Metals (Ref. 4), p. 35, Table 2.1 .

${ }^{25}$ D. Bedeaux and P. Mazur, Physica (Amsterdam) 73, 431 (1974); I. M. de Schepper and M. H. Ernst, Physica A 98, 189 (1979); P. Verkerk, J. H. Builtjes, and I. M. de Schepper, Phys. Rev. A 31, 1731 (1985).

${ }^{26}$ M. Canales and J. Padro, Phys. Rev. E 63, 011207 (2000).

${ }^{27}$ S. Singh and K. Tankeshwar, Phys. Rev. E 67, 012201 (2003); T. Scopigno and G. Ruocco, ibid. 70, 013201 (2004); S. Singh and K. Tankeshwar, ibid. 70, 013202 (2004).

${ }^{28}$ T. Bryk and I. Mryglod, Phys. Rev. E 64, 032202 (2001).

${ }^{29}$ L. D. Landau and I. M. Lifshits, Fluid Mechanics (Pergamon, Oxford, UK, 1987), Chap. 7, p. 304.

${ }^{30}$ S. M. Mujibur Rahman and L. B. Bhuiyan, Phys. Rev. B 33, 7243 (1986); K. K. Mon, Phys. Rev. E 63, 061203 (2001); Y. N. Zhang, L. Wang, S. Morioka, and W. M. Wang, Phys. Rev. B 75, 014106 (2007).

${ }^{31}$ T. Bryk and I. Mryglod, Phys. Rev. E 63, 051202 (2001); J. Phys.: Condens. Matter 13, 1343 (2001).

${ }^{32}$ D. L. Price, M. L. Saboungi, and F. J. Bermejo, Rep. Prog. Phys. 66, 407 (2003).

${ }^{33}$ S. Krishnan, K. J. Yugawa, and P. C. Nordine, Phys. Rev. B 55, 8201 (1997).

${ }^{34}$ A. H. Said, H. Sinn, A. Alatas, C. A. Burns, D. L. Price, M. L. Saboungi, and W. Schirmacher, Phys. Rev. B 74, 172202 (2006). 